\title{
Evaluation of post-antibiotic effect in Gram-negative and Gram-positive bacteria
}

\author{
Elisa Tavella, Alberto Palenzona, Eugenio A. Debbia, Anna Marchese, Simone Cagnacci \\ Sez. Microbiology- DISCAT, University of Genova. \\ Largo Rosanna Benzi 10 - 16132 Genova
}

Key Words: post-antibiotic effect, Gram-negative cocci, Gram-positive rods

\section{Valutazione dell'effetto post-antibiotico in batteri Gram-negativi e Gram-positivi}

\section{SUMMARY}

Although the postantibiotic effect (PAE) is a well recognized phenomenon, the mechanism by which it is induced has not fully elucidated yet. It has been suggested that PAE is the time required by bacteria to synthesize proteins or mRNA characterized by a short half-life that are consumed during antibiotic treatment. This phenomenon is widely studied on Gram-positive cocci and Gram-negative rods, while information about Gram-positive rods and Gram-negative cocci are scanty. To gain new insights on the PAE, this study was addressed to evaluated the time required by Moraxella catarrhalis and Lactobacillus planctarum to resume their physiological growth rate after exposure to various antibiotics.

Methods PAE was estimated in accordance with the method of Craig and Gudmundsson using the following drugs: penicillin, piperacillin-tazobactam, cefalotin, ceftazidime, imipenem, ciprofloxacin, gentamycin and azithromycin. Log-phase bacteria were exposed to drug at a concentration corresponding to 4 times the MIC value for $\mathrm{I}$. The drug was inactivated by I:I000 dilution. Bacterial counts were determined at time zero, immediately after drug dilution, and at each hour after removal for $6-7 \mathrm{~h}$ by a pour-plate technique. The PAE was defined as the difference in time required by test and control cultures to increase by I log in CFU number.

Results All drugs tested induced a PAE on the strains studied. M. catarrhalis registered PAE values ranging between 0,5 (gentamycin) and 2 (ceftazidime, imipenem and azithromycin). With respect to L. plantarum a PAE between 0,8 (cefalotin) and 3 hours (ciprofloxacin) were detected.

Conclusion. These findings demonstrated that all the drugs tested were able to induce a PAE on the strains tested. This observation differs from that observed on Gram-negative rods characterised by negative PAE values induced by penicillins and cephalosporins. This results might reflect the different target of these compounds on these Gram-positive rods or the different affinity of the antibiotics to several PBPs that required more time to restore the physiological fitness of the bacterial cell.

Received February 12, 2007

Accepted April 16, 2007

\section{INTRODUZIONE}

Il livello dei farmaci antibatterici, dopo aver raggiunto e mantenuto concentrazioni anche superiori alle minime inibenti (MIC) nei distretti dell'organismo sedi dei processi infettivi, scende a valori inferiori (sub-MIC) o si azzera completamente in conseguenza del normale processo di eliminazione da parte degli organi emuntori.

La rapida rimozione del farmaco, d'altra parte, fa sì che i microorganismi siano esposti per un tempo limitato all'antibiotico. Tale cinetica produce effetti metabolici che a volte i batteri sopravvissuti non riescono a superare se non dopo diverse ore dal momento del contatto con l'agente antibatterico. Il periodo di tempo necessario ad un microorganismo per riprendere il tasso di crescita normale, dopo breve esposizione ad un farmaco, è denominato effetto post-antibiotico (PAE) (6). Questo fenomeno ha assunto particolare rilevanza perchè è stata osservata una buona correlazione tra $\mathrm{i}$ dati ottenuti in vitro e quelli verificati in vivo (6). In dettaglio, il PAE è consistente nei Grampositivi trattati con tutti i più comuni farmaci antibatterici, indipendente dal loro meccanismo d'azione, mentre nei Gram-negativi non si evidenzia, in genere, con i ß-lattamici $(5-6,13)$.

E noto che il turnover dei componenti batterici è una risposta fisiologica alle diverse esigenze cellulari, in cui la degradazione enzimatica degli mRNA e la proteolisi regolate dai prodotti di

\section{Corresponding author: Simone Cagnacci}

Sez. Microbiology- DISCAT, University of Genova. - Largo Rosanna Benzi 10 - 16132 Genova

Tel.: 0103538998 - Fax: 0103537698 - E-mail: simone.cagnacci@unige.it 
diversi geni, giocano un ruolo chiave $(11,15)$. L'effetto post-antibiotico quindi potrebbe essere dovuto alla necessità dei batteri di sintetizzare quelle proteine e quegli mRNA, caratterizzati da una breve vita, e consumatisi durante il trattamento antibiotico. Studi condotti sulla base di queste ipotesi e in varie condizioni sperimentali hanno dato interessanti indicazioni. In particolare, risospendendo in soluzione salina tamponata i batteri, è stata creata una situazione di batteriostasi, simile a quella che si ottiene trattando con cloramfenicolo, i germi hanno mostrato in questo caso una latenza nella ripresa della crescita pari a quelli che si ottiene esponendoli a tale antibiotico (10).

Durante il periodo di crescita influenzato dal PAE i microrganismi risultano più resistenti all'attività letale degli antibiotici, mentre appaiono più sensibili a quella dei leucociti polimorfonucleati (14).

La presenza o l'assenza di PAE può suggerire una somministrazione continua o discontinua di un antimicrobico, in modo tale da garantire nel tempo una soppressione della crescita dei microrganismi, infatti mentre per le combinazioni antibiotico/microrganismo che non presentano PAE diventa necessaria una somministrazione continua del farmaco, per quelle a cui corrispondono significativi valori di effetto postantibiotico può essere indicata una somministrazione discontinua $(2,5,8)$.

Pur essendo stato valutato essenzialmente in Gram-positivi di forma coccoide (enterococchi, stafilococchi e streptococchi) o in bastoncini Gram-negativi (Enterobacteriaceae e non fermentanti) con tutte le classi di farmaci, il PAE non è mai stato studiato, salvo rare eccezioni $(1,7)$, nei cocchi Gram-negativi e nei bastoncini Grampositivi. Per questo motivo lo scopo di questo studio è stato quello di valutare l'induzione di effetto post antibiotico da parte di diverse classi di antibiotici su questi ultimi ceppi.

\section{MATERIALI E METODI \\ Ceppi utilizzati}

I ceppi utilizzati (M. catarrhalis e L. planctarum) sono stati scelti in rappresentanza di cocchi Gram-negativi e bastoncini Gram-positivi. $M$. catarrhalis, di isolamento clinico, sensibile a tutti gli antibiotici con la sola eccezione dell'ampicillina, è stato coltivato e mantenuto su MuellerHinton agar. Lactobacillus plantarum P17630, dono della ditta Scharper S.p.A. (Milano), è stato isolato e coltivato su terreno MRS (MercK, Milano) contenente vancomicina $12 \mathrm{mg} / \mathrm{L}$ (MRS V12) e Rogosa (Merck) ma modificato come segue (in $\mathrm{g} / \mathrm{L}$ ): peptone 10 , yeast extract 5 , riboso 25 , fosfato monobasico di potassio 6 , citrato d'ammonio 2, Tween 801 , acetato di sodio 15 , solfato di magnesio 0.575 , solfato ferroso 0.034 , solfato di manganese 0.12 , solidificati quando necessario da $15 \mathrm{~g} / \mathrm{L}$ di agar-agar (L. Morelli, Università Cattolica, Piacenza, comunicazione personale). Questo terreno è risultato fortemente selettivo per $L$. plantarum $\mathrm{P} 17630$. Il ceppo è stato in parte caratterizzato e presenta resistenza intrinseca a vancomicina e teicoplanina, clindamicina, gentamicina, fosfomicina, norfloxacina e metronidazolo (9).

\section{Antibiotici}

Gli antibiotici utilizzati in questo studio sono stati: penicillina, piperacillina-tazobactam, cefalotina, ceftazidime, imipenem, ciprofloxacina, gentamicina e azitromicina, tutti ottenuti da fonti commerciali. Le soluzioni madri sono state preparate in occasione dell'esecuzione di ogni singolo esperimento a partire dalle polveri standard, sciolte nel solvente indicato dal fornitore, sino a ottenere una concentrazione finale di $1 \mathrm{mg} / \mathrm{ml}$.

\section{Determinazione delle minime concentrazioni inibenti}

Le minime concentrazioni inibenti (MIC) di ogni antibiotico nei confronti di tutti i ceppi utilizzati negli esperimenti, sono state determinate mediante la tecnica della microdiluizione in brodo (Mueller-Hinton) secondo le linee guida suggerite da CLSI (Clinical and Laboratory Standard Institute, 2005) (4).

\section{Determinazione del PAE}

L'effetto post-antibiotico è stato valutato seguendo la metodica descritta da Craig e Gudmundsson (6). I batteri, cresciuti sino al raggiungimento della fase di crescita logaritmica, sono stati diluiti in Mueller-Hinton (MH) brodo sino ad una concentrazione di $10^{6}-10^{7} \mathrm{CFU} / \mathrm{ml}$ e suddivisi in due aliquote. La soluzione acquosa dell'antibiotico, è stata aggiunta ad una concentrazione pari a quattro volte il valore della MIC ad una soltanto delle due parti della brodocoltura diluita. Dopo un'ora di incubazione in agitazione a $37^{\circ} \mathrm{C}$ in un bagno termostatato, l'antibiotico è stato eliminato diluendo 1:1000 il campione trattato in terreno preriscaldato $\left(37^{\circ} \mathrm{C}\right)$ privo di antibiotico. Anche il campione non trattato è stato sottoposto alla medesima diluizione ed entrambe le brodocolture sono state incubate nuovamente. Le conte batteriche sono state determinate diluendo adeguatamente aliquote dei due campioni (prelevate a tempo zero, immediatamente dopo l'eliminazione dell'antibiotico e dopo ogni ora, per un totale di sette ore) e successivamente seminate su piastre di $\mathrm{MH}$-agar.

Il PAE è stato valutato come la differenza tra il tempo richiesto dal ceppo trattato $(\mathrm{T})$ e quello impiegato dal ceppo non trattato $(\mathrm{C})$ per avere un aumento pari ad $1 \log$ del numero delle unità for- 
manti colonia per millilitro $(\mathrm{CFU} / \mathrm{ml})$, utilizzando l'equazione standard sottoriportata:

$\mathrm{PAE}=\mathrm{T}-\mathrm{C}$, dove gli intervalli di tempo $\mathrm{T}$ e $\mathrm{C}$ sono espressi in ore e calcolati dai disegni delle curve di crescita del ceppo trattato e di quello non trattato.

\section{RISULTATI}

In esperimenti preliminari il PAE è stato valutato utilizzando la metodica usuale, descritta nella sezione Materiali e Metodi, che prevedeva l'impiego di una concentrazione di antibiotico corrispondente a 4XMIC. Le stime del numero dei sopravvissuti, tuttavia, hanno evidenziato che in queste condizioni sperimentali i ceppi batterici non risentivano della presenza degli antibiotici. Le condizioni sperimentali di determinazione della MIC e della valutazione del PAE differiscono, infatti, per alcuni parametri come ad esempio l'inoculo. In particolare il primo saggio stabilisce una concentrazione batterica di $5 \times 10^{5} \mathrm{CFU} / \mathrm{ml}$ mentre il numero dei batteri utilizzati per il PAE non può essere inferiore a $10^{7} \mathrm{CFU} / \mathrm{ml}$, in quanto è prevista una mortalità di almeno il $90 \%$ o una stasi della crescita della popolazione iniziale in seguito al trattamento con antibiotico e, una diluizione di 1:1000 che viene adottata per ridurre il farmaco ad un livello non più efficace per inibire la proliferazione dei batteri. Tutti eventi che portano la consistenza numerica dei germi sopravvissuti al limite della possibilità della tecnica di evidenziare un numero di $\mathrm{CFU} / \mathrm{ml}$ attendibile. Utilizzando un inoculo di $10^{7} \mathrm{CFU} / \mathrm{ml}$ per stimare il PAE, tutti i valori di MIC determinati con la tecnica usuale $\left(5 \times 10^{5} \mathrm{CFU} / \mathrm{ml}\right)$ non corrispondevano più ad una dose capace di inibire la crescita batterica. Pertanto i valori delle MIC sono stati determinate utilizzando un inoculo pari a $10^{7}$ $\mathrm{CFU} / \mathrm{ml}$ partendo da una coltura di $10 \mathrm{ml}$ incubata in ambiente ossigenato mediante agitazione, cioè, nelle stesse condizioni sperimentali in cui si valuta il PAE. I nuovi valori di MIC ottenuti hanno dimostrato aumenti da 2 a 4 volte il livello registrato con le tecniche tradizionali. Le nuove concentrazioni di MIC, che sono state quadruplicate per rispettare la metodica del PAE, hanno tuttavia in alcuni casi mostrato cadute della carica batterica iniziale di oltre il $99 \%$ che, dopo diluizione, ha reso la conta dei sopravvissuti molto difficoltosa a causa del loro esiguo numero, spesso inferiore a $10^{3} \mathrm{CFU} / \mathrm{ml}$, che è il limite mimino della tecnica che accerta le CFU/ml per avere dati riproducibili. Sono state pertanto determinate per ogni combinazione antibiotico/microrganismo i valori di MIC che rispettavano quelli ottenuti con tecnica PAE e la corrispondente dose indicata nella metodica senza superare una mortalità iniziale del $99 \%$.

La concentrazione di farmaco adottata definitivamente è quella riportata in tabella 1 che indica il valore di MIC ed il multiplo utilizzato nell'esperimento.

Come mostrato in tabella 1 , tutti gli antibiotici saggiati hanno indotto effetto post-antibiotico su entrambe le specie batteriche. In particolare con M. catarrhalis il valore di PAE registrato oscillava tra 0.5 (gentamicina) e 2 ore (ceftazidime, imipenem e azitromicina).

Nei confronti di L. plantarum è stata osservata un'induzione di PAE tra 0.8 (cefalotina) e 3 ore (ciprofloxacina).

Il grafico 1 riporta due esempi di determinazione del PAE in M. catarrhalis (A) con imipenem e $L$. plantarum (B) con ciprofloxacina. Prendendo come esempio il grafico $1 \mathrm{~A}$, le frecce indicano i punti in cui le curve dei trattati e dei controlli hanno segnato un aumento di 1 Log $\mathrm{CFU} / \mathrm{ml}$ rispetto al punto di partenza. La prima freccia indica la concentrazione iniziale dei batteri che per il controllo corrisponde a $5 \times 10^{4} \mathrm{CFU} / \mathrm{ml}$. A questo valore corrisponde sull'asse delle ascisse un tempo di un'ora. Quando il controllo raggiun-

Tabella. I. Effetto post-antibiotico indotto da diversi antibiotici in M. catarrhalis, e L. plantarum

\begin{tabular}{|c|c|c|c|c|}
\hline Ceppo & Antibiotico & $\begin{array}{c}\text { MIC } \\
(\mathrm{mg} / \mathrm{L})\end{array}$ & $\begin{array}{c}\text { Dose } \\
\text { usata } \\
\text { XMIC }\end{array}$ & $\begin{array}{l}\text { PAE } \\
\text { (ore) }\end{array}$ \\
\hline \multicolumn{5}{|c|}{ M. catarrhalis } \\
\hline & piperacillina-tazobactam & 0.125 & $\mathrm{I}$ & $1.5 \pm 0.4$ \\
\hline & ceftazidime & 0.06 & 2 & $2 \pm 0.6$ \\
\hline & imipenem & 0.03 & I & $2 \pm 0.7$ \\
\hline & ciprofloxacina & 0.016 & $\mathrm{I}$ & $1.5 \pm 0.4$ \\
\hline & gentamicina & 1 & 2 & $0.5 \pm 0.2$ \\
\hline & azitromicina & 0.06 & 2 & $2 \pm 0.5$ \\
\hline \multicolumn{5}{|c|}{ L. plantarum } \\
\hline & penicillina & 0.25 & 2 & $I \pm 0.3$ \\
\hline & cefalotina & 2 & 2 & $0.8 \pm 0.3$ \\
\hline & imipenem & $\mathrm{I}$ & $\mathrm{I}$ & $1.5 \pm 0.6$ \\
\hline & ciprofloxacina & 0.25 & 2 & $3 \pm 0.6$ \\
\hline & azitromicina & 2 & 2 & $1.6 \pm 0.4$ \\
\hline
\end{tabular}



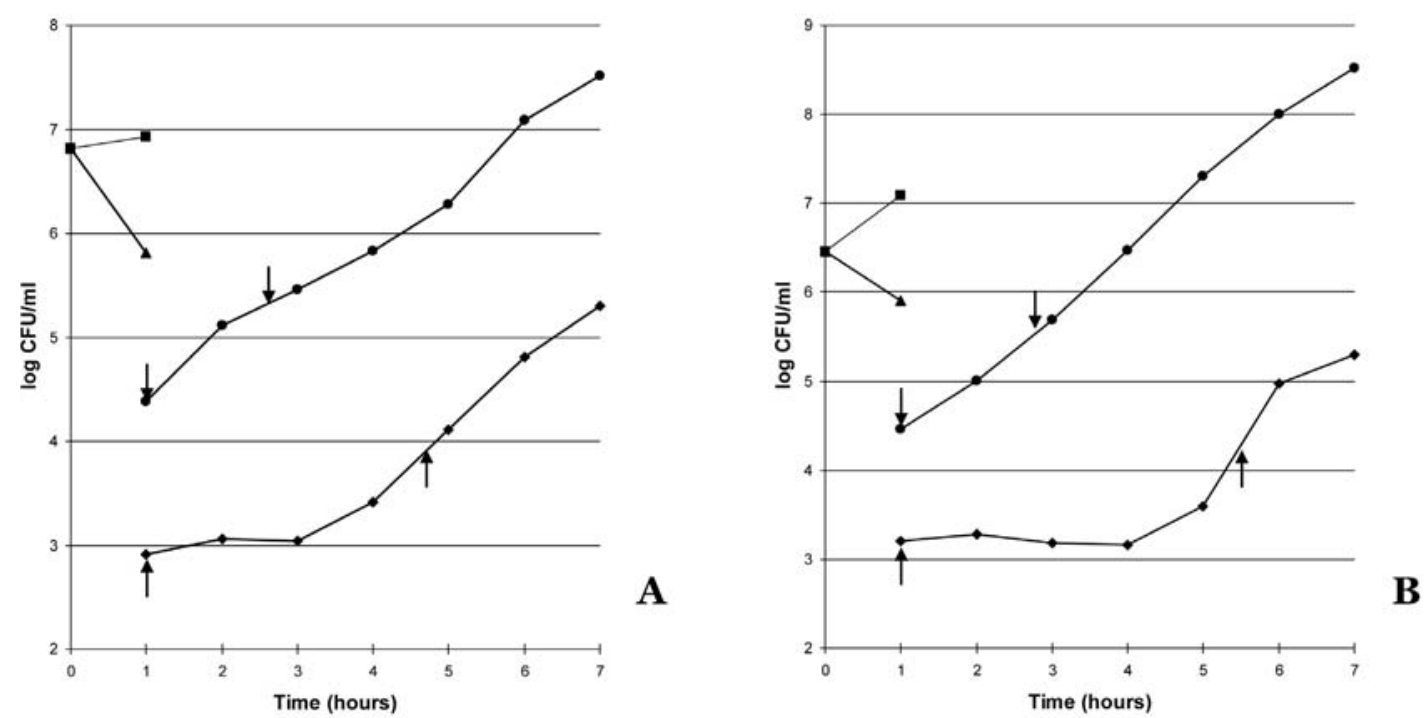

Grafico I. Effetto post-antibiotico indotto in M. catarrahalis esposta per I ora a imipenem e in L. plantarum trattato con ciprofloxacin; Controllo, $(\bullet)$ prima e dopo $(\bullet)$ diluizione I:I000 • trattato $(\mathbf{\Delta})(\diamond)$ nelle medesime condizioni (Vedi testo per $i$ dettagli).

ge una concentrazione di $5 \times 10^{5} \mathrm{CFU} / \mathrm{ml}$, come indicato dalla seconda freccia, il tempo trascorso come riportato sull'asse delle ascisse corrisponde al valore di 2.6 ore. Quindi il controllo per aumentare di un Log ha impiegato 1.6 ore $(2.6-$ 1 ore). Facendo lo stesso conteggio per il trattato, partendo da una concentrazione iniziale di $9.2 \mathrm{x}$ $10^{2} \mathrm{CFU} / \mathrm{ml}$ si arriva alla concentrazione di $9.2 \mathrm{x}$ $10^{3} \mathrm{CFU} / \mathrm{ml}$ dopo 3.75 ore $(4.75-1$ ore $)$. Quindi la differenza in termini di tempo per aumentare di un Log tra trattato e controllo corrisponde a 2.15 ore $(3.75-1.6$ ore $)$ che è il valore dell' effetto post-antibiotico.

\section{CONCLUSIONI}

La scelta di un antibiotico per la profilassi antimicrobica è basata su parametri microbiologici, farmacocinetici, chimici e farmacodinamici. La conoscenza dei parametri farmacodinamici quali l'attività battericida, l'azione nei confronti dei fattori di virulenza batterici e l'effetto post-antibiotico permette una scelta mirata dell'antibiotico da somministrare e l'ottimizzazione della sua posologia (5).

La possibilità di estendere gli intervalli tra le successive somministrazioni di un antibiotico, in virtù della presenza di un effetto post-antibiotico comporta una serie di vantaggi tra cui: una minore tossicità, una maggiore compliance ed una riduzione dei costi.

La valutazione di un effetto post-antibiotico amplia quindi il concetto di attività antibatterica di un antimicrobico, altrimenti limitato alla determinazione della minima concentrazione inibente o battericida del medesimo.

I dati ottenuti dopo aver trattato M. catarralis, e
L. plantarum con diversi antibiotici indicano che la forma dei batteri non appare avere un ruolo diretto nell'induzione di PAE, infatti in tutti è stato registrato un effetto post-antibiotico. Queste osservazione appaiono in linea con quanto riportato per Bacillus anthracis indicando che nei bastoncini Gram-positivi, a differenza di quelli Gram-negativi, le penicilline e le cefalosporine generano PAE.

Il meccanismo alla base del PAE rimane al momento ancora in gran parte da chiarire benché il fenomeno sia noto da lungo tempo (3) e siano ormai state formulate diverse ipotesi. Tuttavia è ragionevole supporre che il necessario ripristino delle funzioni fisiologiche danneggiate dalla presenza dell'antibiotico giochi un ruolo predominante rispetto alla forma batterica nel recupero del normale tasso di crescita al termine del trattamento antimicrobico $(10,12,16)$.

Nel loro insieme questi dati stimolano alcune considerazioni. Il bersaglio cellulare appare influire più decisamente sull'induzione del PAE. Nei bastoncini Gram-negativi il blocco della PBP-3 da parte di penicilline, cefalosporine e aztreonam (7-8) non compromette altra funzione cellulare che la formazione del setto, identificabile anche al microscopio con la formazione di filamenti. Quando il farmaco viene allontanato il processo di divisione si avvia immediatamente e il numero dei batteri che costituisce il filamento viene subito ristabilito, non determinando alcun rallentamento del tasso di crescita.

Nei bastoncini Gram-positivi non è ancora noto il bersaglio dei ß-lattamici e le alterazioni della morfologia nonostante la presenza di forme filamentose (dati non riportati) potrebbe suggerire un 
simile comportamento. In definitiva in infezioni sostenute da questi patogeni Gram-positivi tutti i farmaci appaiono indurre PAE indipendentemente dalla forma batterica con possibili benefici terapeutici.

\section{BIBLIOGRAFIA}

1. Athamna A, Athamna M, Medlej B, Bast DJ, Rubinstein E. In vitro post-antibiotic effect of fluoroquinolones, macrolides, ß-lactams, tetyracyclines, vancomycin, clindamycin, linezolid, chloramphenicol, quinupristin/dalfopristin and rifampicin on Bacillus anthracis. J Antimicrob Chemother 2004; 53: 609-15.

2. Beam TR, DN Gilbert, CM Kunin. General guidelines for the clinical evaluation of anti-infective drug products. Clin Infect Dis, 1992; 15(Suppl): S5-S32.

3. Bigger JW. The bactericidal action of penicillin on Streptococcus pyogenes. Ir. J Med Sci 1944; 227: 53368.

4. Clinical and Laboratory Standards Institute. Performance Standard for Antimicrobial Susceptibility Tests; Fifteenth Informational Supplement. M2-A8. and Supplement M100-S15, Wayne, PA, 2005.

5. Craig WA. Pharmacokinetic/pharmacodynamic parameters: rationale for antimicrobial dosing of mice and men. Clin Infect Dis 1998; 26: 1-12.

6. Craig WA, Gudmundsson S. Postantibiotic Effect. In V Lorian (ed.), Antibiotics in laboratory medicine, 4th ed., William and Wilkins, Baltimore, Md 1996; 296329.

7. Debbia EA, Molinari G, Paglia P, Schito GC. Postantibiotic effect of azitromycin on respiratory tract pathogens. Drug Expl Clin Res XVI 1990; (12): 6159.

8. Debbia EA, Paglia P, Pesce A, Schito GC. Importanza della Microbiologia nella scelta delle dosi dei farmaci antibatterici. Riv Inf Ped 1989; 2, S.3-S.7.

9. Dho G, Marchese A, Debbia EA. Caratteristiche microbiologiche di Lactobacillus plantarum p17630 contenuto in un preparato probiotico per uso vaginale. GIMMOC 2003; VII: 102-8.

10. Dolcino M, Zoratti A, Debbia EA, Schito GC, Marchese A. Post antibiotic effect and delay of regrowth in strains carrying mutations that save proteins or RNA. Antimicrob. Agents Chemother 2002; 46: 4022-5.

11. Gennis RB, Stewart V. Respiration In F. C. Neidhardt (ed.), E. coli and S. typhimurium: cellular and molecolar biology. American Society for Microbiology, Washington, D.C. 1996; 217-61.

12. Li RC, Lee SL, Kong CH. Correlation between bactericidal activity and postantibiotic effect for five antibiotics with different mechanism of action. J Antimicrob Chemother 1997; 40: 39-45.

13. MacKenzie FM, Gould IM. The post-antibiotic effect. J Antimicrob Chemoter 1993; 32: 519-37.
14. McDonald PI, Wetherall BL, Pruul H. Postantibiotic leukocyte enhancement: increased susceptibility of bacteria pretreated with antibiotics to activity of leukocytes. Rev Infect Dis 1981; 3: 38.

15. Mosteller RD, Goldstein RV, Nishimoto KR. Metabolism of individual proteins in exponentially growing E. coli. J Biol Chem 1980; 255: 2524-32.

16. Stubbings W, Bostock J, Ingham E, Chopra I. Mechanismof the post-antibiotic effects induced by rifampicina and gentamicin in Escherichia coli. J Antimicrob Chemother 2006; 58: 444-8. 